\title{
Remote Sensing Concepts and Their Applicability in REDD+ Monitoring
}

\author{
Timo Tokola ${ }^{1}$
}

Published online: 13 October 2015

(C) Springer International Publishing AG 2015

\begin{abstract}
The Tier approach introduced by the ntergovernmental Panel on Climate Change (IPCC) has been used to describe levels of methodological quality and complexity in the measurement reporting and verification (MRV) assessment system. Although guidelines do not explicitly state accuracy requirements, it has been asserted in previous studies that remote sensing missions should entail biomass errors that remain within $20 \%$ of stand level estimates. The biomass of the target forest can be almost directly measured using LiDAR techniques when other sensors have rather low capacity to penetrate through canopy and response signals are not too noisy to detect changes in closed forests. Optical or radar satellite data, in relative terms, are only a tenth of the price of LiDAR. Taking into account cost-effectiveness within a tropical context, it is proposed that Landsat-like data can be useful for obtaining land cover and economical estimates for large areas. Typically, the role of airborne LiDAR is to provide ground truth type of information covering large areas. The reducing emissions from deforestation and forest degradation measurement, reporting and verification (REDD MRV) will need good ground sample for basal area and canopy cover change detection. LiDAR will be also feasible for forest management planning maps and planning operational REDD activities, but cost-effective mapping of carbon stocks for regional- and national-scale assessments will rely on a combination of satellite imagery
\end{abstract}

This article is part of the Topical Collection on Remote Sensing

Timo Tokola

timo.tokola@uef.fi

1 School of Forest Sciences, University of Eastern Finland, Joensuu Borealis 363, P.O. Box 111, 80101 Joensuu, Finland and ground-based inventory samples. The distribution of tree species and different forest types need to be measured from the field and information can be generalised using satellite data with $1-30-\mathrm{m}$ resolution. The global scale maps with the ground resolution $100 \mathrm{~m}-1 \mathrm{~km}$ are mainly for visualisation purposes and can be used to extrapolate distribution of information collected by $1-30-\mathrm{m}$ remote sensing sensors and field plots.

Keywords Forests $\cdot$ Remote sensing $\cdot$ REDD MRV (reducing emissions from deforestation and forest degradation measurement, reporting and verification) $\cdot$ Biomass

\section{Introduction}

One economic incentive mechanism for reducing greenhouse gas emissions from deforestation and forest degradation in developing countries is that commonly referred to as reducing emissions from deforestation and forest degradation (REDD+). Establishing a robust and transparent system to support local and national measurement, reporting and verification (MRV) of biomass resources and forestry activities are required for the successful implementation of a REDD+ regime. These MRV requirements are fixed by UNFCCC and need to be fulfilled by the national or local monitoring system over each target forest area. Without precise and credible MRV systems, it will not be possible to compensate countries or communities efficiently, effectively and equitably for reducing their emissions.

At the same time, the quantification of heterogeneity in forest areas has long been an objective of forest inventories. Heterogeneity and the methodology required for assessing it are highly dependent on scale. The spatial 
and temporal variation of a given property will often depend on the spatial and temporal scale at which that property was sampled, and on the size of the minimum mapping unit. The information levels used in forestry reporting are hierarchically divided into: (1) tree level, (2) stand level, (3) farm level, (4) region level and (5) country level [1], and data collection is normally based on measured sample units or subjective field observations derived from the reporting units. The spatial pattern of these reporting units can be mapped using remote sensing techniques or field observations. Permanently documented practices and local biometric models are also needed in order to establish a reliable monitoring system.

The procedure for estimating above-ground (AG) carbon employs certain specific coefficients and variables, as described below:

$\begin{array}{ll}\text { Stem biomass } & =\text { Stem volume } \times \text { Wood gravity } / \text { density factors } \\ \text { Tree biomass A G } & =\text { Stem biomass } \times \text { Biomass expansion factor } \\ \text { Tree carbon A G } & =\text { Tree biomass } \times \text { Carbon factor } \\ \text { Forest area carbon AG } & =\text { Area } x \text { basal area } \times \text { Tree carbon A G }\end{array}$

Stem volume/size is typically a function of height, tree diameter and species. Tree heights can be measured using specific remote sensing sensors like LiDAR, interferometric synthetic aperture radar (InSAR), or by photogrammetry, while tree diameter requires field measurements, and species can partly be estimated in boreal areas using near infrared observations of crown shape, e.g. [2]. Although homogenous forest stand can be mapped with optical data, the species recognition is very difficult task in mixed-species stands and tropical areas. Several researchers, e.g. [3, 4] have used hyperspectral data to assess species and found the low changes to detect specific species. Wood gravity factors are in turn highly species-specific (Table 1) and dependent on the status of the forest ecosystem, while biomass expansion factor (BEF) describes stem forms and the allocation of the biomass to components. BEF is an easy, reliable method for estimating biomass components when the trees are uniform and do not have a complex branch structure, but this is very rarely the case in the tropics. BEF and the carbon conversion factor can be unique for each tree species, or else the results can be computed by using the same factor for all species. In addition to tree height, basal area and the area of the target forest can be almost directly measured using LiDAR techniques, e.g. [5••, $6 \cdot \bullet$, when other sensors have rather low capacity to penetrate through canopy and response signals are not too noisy. Localscale field measurements are integral to MRV, and forest biomass should be estimated using field measurements by

Table 1 Statistics for tree species wood density factors $\left(t / \mathrm{m}^{3}\right)$ in specific tropical areas as listed by the FAO [13]

\begin{tabular}{llllll}
\hline Area & $N$ & Mean & Std & Min & Max \\
\hline Tropical Africa & 282 & 0.581 & 0.144 & 0.200 & 0.930 \\
Tropical Asia & 428 & 0.564 & 0.153 & 0.210 & 1.040 \\
Tropical America & 470 & 0.594 & 0.164 & 0.120 & 1.080 \\
\hline
\end{tabular}

applying allometric models and factors assessed by sampling and weighing dried vegetation components. Even so, an international default values have been given by [7], but very often rough estimates (e.g. 0.50) have been employed in the calculations required for remote sensing-based applications without detailed species separation. It suggests that these represent an averaging method and should not be encouraged for use in assessing species variability. Remote sensing has nevertheless been successfully used for mapping total woody vegetation cover, biomass and other vegetation parameters such as basal area and stand age, e.g. [8-10]. Many radar techniques have been tested for global mapping over the last few decades (see [40]), but still many organisation rely on optical remote sensing, which can indirectly indicate the amount of biomass present when Landsat ETM type of data is used from the right season $[11 \bullet \cdot$.

The Tier approach introduced by the IPCC has been used to describe levels of methodological quality and complexity in the MRV assessment system. Tier-1 applies when no local field data or models are available. Tier-2 has been proposed when species-specific volume equations exist but there are no local biomass conversion or expansion factors [7]. Tier-3 applies when a local speciesspecific biomass equation is available for calculating either the total or partial biomass and organised data collection with permanent sample plots has been implemented for monitoring purposes.

Use of the Tier-2 approach is severely limited by the poor accuracy of BEF estimates [12]. The BEFs used in the [7] were developed by [13] for tropical rain forests and were designed to convert industrial volumes into biomass values; a 10-cm-tree diameter limit was also applied to the modelling approach. In dry tropical forests, the merchantable volume is not limited to the trunk but can also include the stump and branches of up to $3 \mathrm{~cm}$ in diameter. Availability of wood gravity values is one limiting factor and source of error, however, and when it is not possible to find a species-specific 
wood gravity estimate, it is preferable to use the average wood gravity for the family or functional group rather than the IPPC average [12].

Although MRV guidelines do not explicitly state accuracy requirements, it has been asserted in previous studies that remote sensing missions should entail biomass errors that remain within $20 \%$ of field estimates and should not exceed $50 \mathrm{Mg} \mathrm{ha}^{-1}$ for a global biomass map at a resolution of 1 ha [12].

\section{State of Forests - Total Estimates and Forest Area Coverage}

Recent reviews of methods used to estimate deforestation have highlighted two means of monitoring large areas of forest: the 'wall-to-wall' approach, or sampling. Point information obtained from sample plots is normally generalised according to the following methods:

1. A predefined sampling scheme for predefined reporting areas or forest stands, in which sets of remote sensing material with different spatial resolutions can be included within the same multi-phase sampling frame, e.g. $[14,15$, $16 \bullet \bullet]$,

2. Interpolation based partly on geographical distance and partly on remote sensing data, as in KNN-type imputation, or alternatively generalisation methods, as used in many national forest mapping systems, e.g. [17-19] or

3. Modelling without any strict sampling design for remote sensing-based segments or forest stands, e.g. [20].

When a multi-phase sampling and design-based frame is employed, we have 'text book' estimates to derive reliability estimates and guarantee verification of the inventory process. Forest inventory is not typically a standard design-based approach, and we need to consider complex model-based estimators $[14,15,16 \bullet \cdot$. When very high resolution remote sensing data are used to impute field data using satellite imagery, additional modelling is needed, and an additional phase will have to be included in the sampling frame. Detailed attributes typically call for an additional phase when the original RS data do not bear any actual relation to the required attribute (e.g. species-specific tree growth).

Satellite imagery-based wall-to-wall approaches [21-23] have been used to map the full extent of a forest area and its changes by means of various sensors. This is typically done using hybrid approaches that combine automated digital segmentation, classification or fully automated techniques [11••, 24-27]. The sampling approach consists of identifying partially representative land cover observations by means of field sample plots or visual interpretation of satellite images in order to estimate the extent of the forest cover and of deforestation over an entire area [28, 29]. The presence of clouds, shadows and haze, and the season of the year, can nevertheless affect the quality and completeness of the data obtained from optical sensors, especially in tropical areas.

\section{State of Forests - Biomass and Vegetation Structure}

Several studies in countries such as India [30], Bolivia and Brazil [31, 32], Malaysia [33] and Costa Rica [34] have pointed to significant correlations between biomass and reflectance measured at different optical wavelengths. The limitation to measuring only the upper part of the canopy results in large uncertainties, however, and the methods used may not be applicable under all conditions [35, 36]. Steininger [31], for example, found the canopy reflectance-biomass relationship to saturate at around $150 \mathrm{Mg} / \mathrm{ha}$, while [37] reported that the above-ground biomass, below-ground biomass and necromass estimates for the large geographical expanse of the Brazilian Amazon could vary from minimum estimates of 78 billion $\mathrm{Mg}$ up to a maximum of 186 billion $\mathrm{Mg}$. Another approach for estimating biomass by remote sensing is based on canopy density [38, 39], as presented on tree cover percentage maps. The SAR-based technologies are rather sensitive to environmental conditions on a per scene basis [40] and have many challenges in worldwide mapping campaigns. Topographic reliefs, precise viewing angles and wet conditions are difficult to control in large area SAR data mosaic processing. Typically, SAR-based biomass estimates have lower precision at same resolution compared to optical data $[41 \bullet \bullet, 42]$.

Grinand et al. [27] concluded that the use of multi-date images is a practical way of reducing classification errors, enabling better characterisation of small complex changes in land cover status. However, small geometric and thematic errors such as confusion between the sub-categories of land cover classes (e.g. two different forest strata or seasonal thicket/dry forest alternation) compromised the true detection of changes. Secondly, new machine-learning algorithms could be used to enhance the interpretation process in the case of complex datasets or land cover patterns [25]. The random forests algorithm was found to improve accuracy [27] and the related visual output when a supplementary training plot was added, and the availability of very high resolution (VHR) images such as the QuickBird/Google Earth images can partially meet this need for information without extensive field surveys. It is also concluded that 10-m-resolution images would greatly improve deforestation monitoring and would also enable the detection of regeneration and degradation $[41 \bullet \bullet, 43]$.

The recent development of very high resolution SAR sensors (e.g. TerraSAR) and processing techniques have increased the expectations towards a feasible SAR 
mapping concept [40]. Saatchi et al. [44] have demonstrated the high sensitivity of P- and L-band airborne SAR observations to above-ground biomass (AGB) $<300 \mathrm{Mg} /$ ha on a one-hectare scale. Still, SAR sensors have mainly been used in experimental and landscape level. Saatchi et al. [34] demonstrated multi-step biomass mapping exercise using a combination of data in situ inventory plots and LiDAR samples of forest structure to estimate carbon storage, plus optical and microwave imagery (1-km resolution) to extrapolate over the landscape. Results were planned to be used for areas exceeding 10,000 ha. Recently, spaceborne LiDAR used together with radargrammetric processing [45] has yielded promising results to provide small area estimates from SAR data, as it allows measurement of the height and vertical structure of a forest. It is by far one of the most accurate options among the various sensors available for estimating biomass on a local scale.

During the last decade, airborne light detection and ranging (LiDAR) has proved able to solve major difficulties by making it possible to measure high-resolution characteristics of forest structure, including height and fine-scale canopy density, or variability in height. One critical feature for forest studies is that the vertical canopy height profile statistics derived from LiDAR have been shown to correlate very well with field-based estimates of vegetation volume, density and structure [6••, 46-48] even under variable tropical conditions. Currently, LiDAR is operationally cost-effective mapping method for detailed forest management purposes or based on sampling, e.g. [10]. The spaceborne sensors needed to use LiDAR approaches for large-scale wall-to-wall mapping and monitoring efforts will not be available before the end of this decade [40].

Although the literature on the estimation of tropical forest attributes, especially species-specific stem volume, which can be used indirectly as a measure of carbon via a biomass expansion factor, is still sparse, the statistical relationships between LiDAR metrics and field measurements of total biomass and stem volume have been studied in tropical forests in various parts of world. Zolkos, Goetz and Dubayah [49] conducted a meta-analysis of reported biomass accuracy estimates using more than 70 refereed papers and a variety of remote sensing platforms (airborne and spaceborne) and sensor types (optical, radar and LiDAR). The study was based on six methodologically different categories of biomass estimation: (i) airborne discrete return LiDAR, (ii) airborne fullwaveform LiDAR, (iii) spaceborne full-waveform LiDAR, (iv) multi-sensor (i.e. LiDAR plus radar or passive optical), (v) radar and (vi) passive optical. Their conclusion was that the mean of the multiple $R^{2}$ was similar for LiDAR and multisensor models $(0.70-0.80)$, while that for the radar and passive optical models was considerably lower $\left(R^{2}=0.50-0.59\right)$. Thus, this comparison of various forest attributes estimated using different sensors also suggests that those based on
LiDAR can be used to predict a wider range of forest attributes with better $R^{2}$ values [49].

An attractive alternative to attempting to map areas wall-towall by aircraft acquisition methods consists of designs that use multi-phase or multi-stage sampling based on Landsattype optical data and limited airborne LiDAR [50]. Strips (flight lines) can be flown and field plots placed within these acquisition boundaries, for example, and they can then be extended to larger areas using additional data sources, [51]. The following observations can be made use of to guide the planning of new multi-phase survey campaigns [49]:

(i) Above-ground biomass models developed from airborne LiDAR metrics are significantly more accurate than those using radar or passive optical data.

(ii) Above-ground biomass models developed from multisensor metrics are more variable than LiDAR-only models, and they do not always improve biomass estimates.

(iii) Model accuracy varies with forest type, species variability and many underlying factors. Even the quality of field measurements can be open to improvement, since complex stem forms are typical in the tropics.

(iv) Modelling errors decline relative to the magnitude of the field biomass with increasing plot size, on account of the averaging effect of a large sampling unit. Small training plots show great variability in surveys, but large numbers of small plots is a more practical solution than a few large plots [52].

Rahlf et al. [53] similarly compared the accuracy of timber volume predictions in a survey area in southern Norway based on four three-dimensional remote sensing datasets: airborne laser scanning (LiDAR), stereo aerial photogrammetry, satellite interferometric synthetic aperture radar (InSAR) based on the TanDEM-X mission and satellite radargrammetry based on the TerraSAR-X mission. They fitted linear-mixed effects models with vegetation height and density metrics obtained from the remote sensing datasets as explanatory variables and used the cross-validated root mean squared error (RMSE) relative to the observed mean as a measure of the goodness-offit. Airborne laser scanning (ALS) provided the most accurate prediction at the plot level, with RMSE $=19 \%$, followed by photogrammetry (31\%), InSAR (42\%) and radargrammetry (44\%). The order of performance of the various methods was the same at the stand level, with RMSE values of $12-23 \%$.

\section{Techniques for Assessing Change in Tropical Forest Areas}

Consistent and accurate detection of tropical forest changes is fundamental to the reliable estimation of greenhouse gas 
emissions and successful implementation of climate mechanisms such as REDD+ [7]. Satellite-based remote sensing at medium spatial resolution $(10-30 \mathrm{~m})$ supported by field observations is an appropriate tool for assessing historical and future changes in forest area and carbon stocks in most tropical countries [54-56].

Following the opening of the Landsat archive, many studies have demonstrated the operational capabilities of optical medium-resolution satellite imagery for detecting changes and trends in forest cover on scales ranging from local to global $[57,58]$, in spite of restrictions imposed on data availability by the frequent cloud cover. Persistent cloud cover inhibits full optical coverage from Landsat-like sensors even when compositing is performed over a period of 1-2 years. A number of optical and C- and L-band radar satellite constellations have provided an additional source of time series data since the mid-1990s, but factors such as wetness differences, atmospheric conditions and the imaging angles of the sensors have quite often caused difficulties in their interpretation.

The available remote sensing-based change detection techniques are mainly based on post-classification, or direct classification of image-to-image change vectors [59••]. Both pixel and object-based approaches have been applied, and it is not immediately obvious which is the optimal one [59*0]. While the object of the image is theoretically the most powerful unit for comparison purposes, scene illumination/atmospheric effects, changes in viewing geometry, scale and the need for identification of small 'sub-area' changes and various segmentation parameters always entail problems that affect the consistency of the results [59••]. Relative pixel-based change vector analysis with subsequent field checks is still a highly valid method for detecting changes [59••]. When using an ordinal logistic regression (OLR) approach and Landsat NDVI time series data, magnitude of the changes as calculated from the differences between the observed and expected values over a given monitoring period has been found to be an essential predictor of small-scale forest disturbances [60••]. This type of method is principally relevant to tropical countries engaged in REDD + for whom data availability and complex forest change dynamics limit the ability to track forest disturbances reliably over time.

It is doubtful whether the losses in biomass per hectare over time due to forest degradation can be accurately quantified using optical or radar remote sensing [27]. The same would appear to be the case for aerial photos and data obtained from low-flying unmanned aircraft (UAVs). Obviously, it depends on the degree of degradation, which is very much basal areaand forest density-related phenomena. The remote sensing field has recently achieved advances in the assessment of AGB by combining different types of optical sensor with LiDAR and/or radar data, as the latter techniques are able to sense and record forest structure metrics (e.g. maximum canopy height) and penetrate both cloud and canopy cover [61,
62]. Such surveys have been made at a national level for relatively small countries (e.g. Panama) with a high degree of accuracy and have allowed quantification of a reference level for forest carbon stocks [61]. This image fusion type of approach exhibits great potential for the future monitoring of stock level changes, provided that data acquisition with the current technology can be rendered sufficiently cost-efficient.

\section{A Case Study in the Planning of a Carbon Inventory: Different Forest Populations and Remote Sensing Alternatives}

The variability in forest attributes directly affects the amount of fieldwork required. When variation is high, more field plots are required, which may be compensated for by a high $R^{2}$ between the field and remote sensing data. The total cost of the fieldwork required for the measurements was estimated during the test inventories, given that the number of plots needed in each reporting are can be determined with reference to the correlation with remote sensing data.

As an example, provincial variation in the Lao PDR was found to be high [63], as seen from the decreasing trend in this and the importance of accurate remote sensing information as illustrated in Fig. 1. As the $R^{2}$ value between the field data and the remote sensing data increases, the need for fieldwork decreases rapidly. The size of the sample plots is also important in this respect, however, for if they are too small, the variation between them will be very high. By contrast, however, the inclusion probability for small areas of large trees will then be so low that relatively large plots are required. It is nevertheless recommended that, where possible, whole plots should fall into the same forest type, so that remote sensing procedures can utilise plot information directly as a ground truth training area. It is shown in Fig. 1 that almost double the number of small field plots is required relative to large plots when there is a poor correlation between the field and remote sensing data.

The cost and quality of remote sensing are normally interconnected. Medium-resolution satellite data are quite cheap per areal unit and an entire country can easily be covered at low cost, whereas VHR satellite data and ALS data are normally used on a sampling basis. Depending on the occurrence of autocorrelation in the forest population, certain types of information can be collected from a single sampling unit. For the comparison of different sets of material, cost estimates can be prepared for a number of scenarios. The required sampling-based number of remote sensing data plots in a $25 \mathrm{~km}^{2}$ area in Laos was determined using autocorrelation values, and the coefficients of determination $\left(R^{2}\right)$ were estimated for land use and biomass based on the different sets of material. The values for biomass in the ALS data were based on pilot data. 


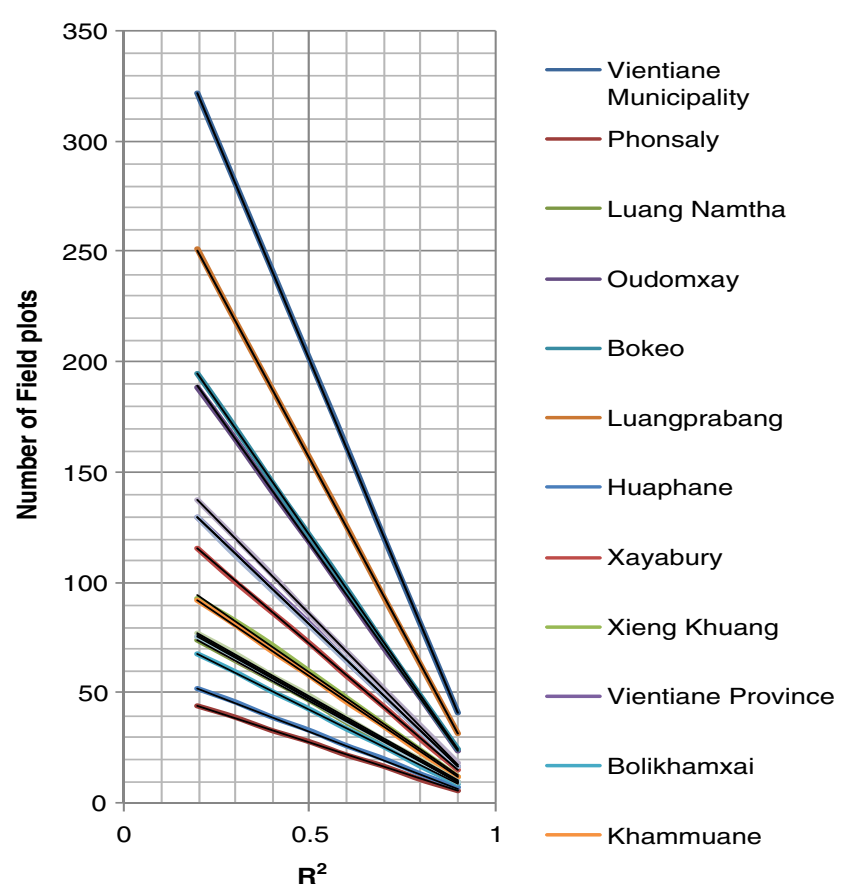

Fig. 1 Comparison of field data needs in different provinces and at different $R^{2}$ levels. When a strong correlation exists between remote sensing and the field data, there is little need for field data even in provinces with wide variations [59••]

The cost of field data must be added to the image data costs in order to estimate the total cost of the inventory system. A medium-resolution image mosaic covering the whole country is normally needed, together with additional VHR/ALS imagery for proper field sampling. Thus, the final system is a combination of full coverage, remote sensing sampling and field plots. According to price assumptions in Laos, the most efficient way was to use ALOS AVNIR data, laser scanning and field data. In this case, field plots were planned for the forest areas only, and additional cost figures and plots were needed for the other land use strata (forest, non-forest, uncertain). However, we need to calculate the number of images needed, availability and field data cost factors always according to the actual situation.

\section{Discussion}

Among the remote sensing approaches reviewed here, it is LiDAR that has demonstrated the most promising performance when estimating stem volume and basal area in a mixed-species tropical forest. Fused wall-to-wall optical data are always needed when large areas are to be covered for mapping purposes. VHR imagery can give a similar response in small-scale mapping, however, so that airborne CIR and LiDAR are both feasible solutions. The availability of the various data sources and their price relations can vary significantly between areas and service providers.
In terms of methodology, it is feasible to extend the popular Scandinavian approaches to the estimation of boreal forest attributes [8] to this tropical context. Among the data sources tested in different parts of world, it was LiDAR that proved to be the most accurate and competent, thus complementing the findings of other investigations focused on tropical areas. Land cover monitoring has been demonstrated in the tropics on several occasions $[24,50]$, but inaccurate LiDAR measurements are still prone to occur in hilly or mountainous regions, with errors extending up to several metres [64]. Furthermore, the observation concerning the effectiveness of hybrid models concurs with that of [65], who, when comparing LiDAR-only and joint LiDAR-radar models, concluded that there was little to be gained from a combination of sensors [6••]. The fusion of satellite imagery, strips of LiDAR data and field plots is a promising approach for the inventory of tropical forests [34]. Airborne LiDARs also enable an accurate direct estimation of the forest canopy cover (CC), and thus a sample of LiDAR strips can be used as reference data for creating CC maps which are based on satellite images [39].

With respect to the cost of data procurement, LiDAR is the most costly despite its low pulse density of $1 \mathrm{pulse} / \mathrm{m}^{2}$, the second most expensive being Airborne CIR. Studies with pulse densities from 25 pulses $/ \mathrm{m}^{2}$ [48] down to about 1.5 pulses $/ \mathrm{m}^{2}$ [45] have indicated that high pulse density is not requisite for estimation of forest biomass. Optical or radar satellite data, in relative terms, are only a tenth of the price of LiDAR. Taking the cost-effectiveness in a tropical context into consideration, Landsat-like data could potentially be useful for obtaining land cover but economical estimates, while LiDAR data provide an alternative that would satisfy a demand for accuracy required in forest management planning and below $40 \%$ at the plot level $[6 \bullet \bullet, 20]$. The needs for accurate remote sensing data are well justified in forest areas with high variability.

Forest variables may be predicted accurately in the case of a tropical forest if the relations used are derived from that site. Predictive relations typically decline in accuracy when they are applied to a region other than that for which they had been developed. Site-specific fieldwork is still important even when considering remote sensing-based inventories in tropical forests. Model-based inference used together with synthetic estimation is a relevant and useful approach for estimation and inference purposes in non-sampled areas [14, 51]. As noted, potential applications include remote tropical and boreal forests for which access is difficult and sampling is either limited or even impossible.

\section{Conclusion and Recommendations}

It may be stated that remote sensing-based applications are definitely key tools for REDD+ MRV monitoring, but we 
still need to safeguard the good practices that are already in use in traditional forest inventories [66]. We still need to implement a sampling frame and design that meets the accuracy demands and satisfies the practical cost constraints. The fieldwork needs to be planned so that it achieves adequate spatial and temporal representation and coverage without any appreciable design bias effects. We should also develop procedures for inaccessible areas, which will normally call for the use of remote sensing tools. Accuracy assessment and the provision of confidence intervals as estimates of the uncertainty involved are aspects that are very often forgotten, because little attention is paid to proper designing of the sampling and fieldwork. All previous forest inventories and spatial images can be used when planning a new data collection methodology. Although free Landsat data are available and we have high quality LiDAR instruments, we need to collect and allocate the field plots carefully, especially in tropical forests where snake-like tree stems are very common, and the tree species have unknown wood density factors.

The role of airborne LiDAR is to provide ground truth type of information from large areas. The REDD MRV will need good ground sample for basal area and canopy cover change detection. LiDAR will be also be feasible for forest management planning maps and planning operational REDD activities, but cost-effective mapping of carbon stocks for regionaland national-scale assessments will rely on a combination of satellite imagery and ground-based inventory samples. The distribution of tree species and different forest types need to be measured from the field, and information can be generalised using satellite data with $1-30 \mathrm{~m}$ resolution. The global scale maps with the ground resolution $100 \mathrm{~m}-1 \mathrm{~km}$ are mainly for visualisation purposes and can be used to extrapolate distribution of information collected by 1-30 m remote sensing sensors and field plots.

The innermost REDD+ concept is still in the realm of forest inventory, and therefore sources of estimate uncertainty actually arise from the same factors that have long been studied by foresters. Standard sampling theory assumes that the quantities entering the estimators are observed without error, but if measurement errors and model related errors cannot be ignored, uncertainty will be underestimated. Although the importance of proper handling of uncertainties was explicitly advocated by IPCC in its good practice guidance for greenhouse gas reporting, it remains unclear how uncertainty assessments should be conducted. McRoberts [67] demonstrated that the advocated type of assessment through confusion matrices is misleading. There is urgent need to improve reliability estimation techniques (e.g. model-based inference) for less ideal forest inventory situations to improve new information services.

\section{Compliance with Ethical Standards}

Conflict of Interest Dr. Tokola has no conflicts of interests to declare.

Human and Animal Rights and Informed Consent This article contains no studies with human or animal subjects performed by the author.

\section{References}

Papers of particular interest, published recently, have been highlighted as:

•- Of major importance

1. Tokola T. Inventory - Spatial Data. In: Encyclopedia of Forest Sciences, Elsevier. 2004;1:s. 508-513.

2. Pant P, Heikkinen V, Hovi A, Korpela I, Hauta-Kasari M, Tokola T. Evaluation of simulated bands in airborne optical sensors for tree species identification. Remote Sens Environ. 2013;138:27-37.

3. Clark ML, Roberts DA, Clark DB. Hyperspectral discrimination of tropical rain forest tree species at leaf to crown scales. Remote Sens Environ. 2005;96:375-98.

4. Dalponte M, Bruzzone L, Gianelle D. Tree species classification in the Southern Alps based on the fusion of very high geometrical resolution multispectral/hyperspectral images and LiDAR data. Remote Sens Environ. 2012;123:258-70.

5.• Næsset E. Predicting forest stand characteristics with airborne scanning laser using a practical two-stage procedure and field data. Remote Sens Environ. 2002;80(1):88-99. Classical paper behind LiDAR concept applied in the different part of the world.

$6 . \bullet$ Hou Z, Xu Q, Tokola T. Use of ALS, Airborne CIR and ALOS AVNIR-2 data for estimating tropical forest attributes in Lao PDR. ISPRS J Photogramm Remote Sens. 2011;66:776-86. Basal area in tropical forest is key variable in MRV of Redd+, and was studied here with different datasets.

7. IPCC. IPCC Guidelines for National Greenhouse Gas Inventories. 2006. Available from: http://www.ipcc-nggip.iges.or.jp/public/ 2006gl/index.html (accessed 21.01.2012).

8. Tokola T, Pitkänen J, Partinen S, Muinonen E. Point accuracy of a non-parametric method in estimation of forest characteristics with different satellite materials. Int J Remote Sens. 1996;17:2333-51.

9. Tomppo E, Haakana M, Katila M, Peräsaari J. Multi-source national forest inventory - methods and applications. Manag For Ecosys 2008;18 2008. ISBN: 978-1-4020-8712-7 (Print) 978-1-40208713-4 (Online).

10. Maltamo M, Næsset E, Vauhkonen J. Forestry applications of airborne laser scanning. Concepts and case studies. Manag For Ecosys. 2014;27 2014. ISBN: 978-94-017-8662-1 (Print) 978-94017-8663-8 (Online).

11.• Brown S. Estimating biomass and biomass change of tropical forests: a primer. (FAO Forestry Paper - 134), A Forest Resources Assessment publication, FAO FORESTRY PAPER 134, FAO Food and Agriculture Organization of the United Nations, Rome, 1997). 1997. This paper introduces different species specific wood density factors needed to provide reliable forest carbon estimates.

12. Henry M, Picard N, Trotta C, Manlay RJ, Valentini R, Bernoux M, et al. Estimating tree biomass of sub-Saharan African forests: a review of available allometric equations. Silva Fennica. 2011;45(3B):477-569. 
13. Hansen MC, Loveland TR. A review of large area monitoring of land cover change using Landsat data. Remote Sens Environ. 2012. doi:10.1016/j.rse.2011.08.024.

14. McRoberts RE, Naesset E, Gobakken T. Estimation for inaccessible and non-sampled forest areas using model-based inference and remotely sensed auxiliary information. Remote Sens Environ. 2014;154(2014):226-33.

15. Magnussen S, Mandallaz D, Breidenbach J, Lanz A, Ginzler C. National forest inventories in the service of small area estimation of stem volume. Can J For Res. 2014;44:1079-90.

16.• Magnussen S, Tomppo E. Model calibration of k-nearest neighbour estimators. Scand J For Res. 2015. Demonstrating sample based concept and need to calibrate results.

17. McRoberts RE. Probability- and model-based approaches to inference for proportion forest using satellite imagery as ancillary data. Remote Sens Environ. 2010;114:017-1025.

18. Tomppo E, Gschwantner T, Lawrence ., \& McRoberts RE. National Forest Inventories. Springer Sci Bus Media. 2009; http://doi.org/10. 1007/978-90-481-3233-1.

19. McRoberts RE, Næsset E, Gobakken T. Optimizing the k-Nearest Neighbors technique for estimating forest aboveground biomass using airborne laser scanning data. Remote Sens Environ. 2015;163:13-22.

20. Hou Z, Xu Q, Nuutinen T, Tokola T. Extraction of remote sensingbased forest management units in tropical forests. Remote Sens Environ. 2013;130:1-10.

21. Harper G, Steininger MK, Tucker CJ, Juhn D, Hawkins F. Fifty years of deforestation and forest fragmentation in Madagascar. Environ Conserv. 2007;34:1-9.

22. Gutman G, Byrnes R, Covington MS, Justice C, Franks S, Headley $\mathrm{R}$. Towards monitoring land-cover and land-use changes at global scale: the global land use survey. Photogramm Eng Remote Sens. 2005;64:6-10.

23. Freidl MA, McIver DK, Hodges JCF, Zhang XY, Muchoney D, Strahler AH, et al. Global land cover mapping from MODIS: algorithms and early results. Remote Sens Environ. 2002;83:287-302.

24. Asner GP, Knapp DE, Balajji A, Paez-Acosta G. Automated mapping of tropical deforestation and forest degradation: CLASlite. 2009. J Appl Remote Sens. 2009;3(1):033543.

25. Huang C, Kim S, Song K, Townshend JRG, Davis P, Alstatt A, et al. Assessment of Paraguay's forest cover change using Landsat observations. Glob Planet Chang. 2009;67:1-12.

26. Potapov PV, Turubanova SA, Hansen MC, Adusei B, Broich M, Alstatt A, et al. Quantifying forest cover loss in Democratic Republic of the Congo, 2000-2010, with Landsat ETM+data. Remote Sens Environ. 2012;122:106-16.

27. Grinand C, Rakotomalala F, Gond V, Vaudry R, Bernoux M, Vieilledent G. Estimating deforestation in tropical humid and dry forests in Madagascar from 2000 to 2010 using multi-date Landsat satellite images and the random forests classifier. Remote Sens Environ. 2013;139(2013):68-80.

28. Rasi R, Bodart C, Stibig H-J, Eva H, Beuchle R, Achard F, et al. An automated approach for segmenting and classifying a large sample of multi-date Landsat imagery for pan-tropical forest monitoring. Remote Sens Environ. 2011;115(12):3659-69.

29. Steininger MK, Godoy F, Harper G. Effects of systematic sampling on satellite estimates of deforestation rates. Environ Res Lett. 2009;4:034015.

30. Roy PS, Ravan SA. Biomass estimation using satellite remote sensing data - an investigation on possible approaches for natural forest. J Biosci. 1996;21:535-61.

31. Steininger MK. Satellite estimation of tropical secondary forest above-ground biomass: data from Brazil and Bolivia. Int $\mathrm{J}$ Remote Sens. 2000;21:1139-57.
32. Lu D, Mausel P, Brondizio E, Moran E. Relationships between forest stand parameters and Landsat TM spectral responses in the Brazilian Amazon Basin. For Ecol Manag. 2004;198:149-67.

33. Phua M-H, Saito H. Estimation of biomass of a mountainous tropical forest using Landsat TM data. Can J Remote Sens. 2003;29(4): 429-40.

34. Saatchi SS, Harris NL, Brown S, Lefsky M, Mitchard ETA, Salas $\mathrm{W}$, et al. Benchmark map of forest carbon stocks in tropical regions across three continents. PNAS. 2011;108(24):9899-904.

35. Anaya JA, Chuvieco E, Palacios-Orueta A. Aboveground biomass assessment in Colombia: a remote sensing approach. For Ecol Manag. 2009;257:1237-46.

36. Foody GM, Boyd DS, Cutler MEJ. Predictive relations of tropical forestbiomass from Landsat TM data and their transferability between regions. Remote Sens Environ. 2003;85:463-74.

37. Houghton RA, Lawrence KT, Hackler JL, Brown S. The spatial distribution of forest biomass in the Brazilian Amazon: a comparison of estimates. Glob Change Biol. 2001;7:731-46.

38. Suganuma H, Abe $Y$, Taniguchi $M$, Tanouchi H, Utsugi H, Kojima $\mathrm{T}$, et al. Stand biomass estimation method by canopy coverage for application to remote sensing in an arid area of Western Australia. For Ecol Manag. 2006;222:75-87.

39. Korhonen L, Ali-Sisto D, Tokola T. Tropical forest canopy cover estimation using satellite imagery and airborne lidar reference data. Silva Fennica. 2015;49((5). article id 1405.

40. Lucas RM, Mitchell AL, Armston J. Measurement of forest aboveground biomass using active and passive remote sensing at large (subnational to global) scales. Curr For Rep. 2015;1(3):162-77.

41.• Hame T, Kilpi J, Ahola HA, Rauste Y, Antropov O, Rautiainen M, et al. Improved mapping of tropical forests with optical and SAR imagery, Part I: forest cover and accuracy assessment using multiresolution data. Sel Top IEEE J Appl Earth Observ Remote Sens. 2013;6(1):74-91. doi:10.1109/JSTARS.2013.2241019. Good comparison of reliability estimated in tropical production forest area.

42. Hame T, Rauste Y, Antropov O, Ahola HA, Kilpi J. Improved mapping of tropical forests with optical and SAR imagery Part II: above ground biomass estimation. Select Top IEEE J Appl Earth Observ Remote Sens. 2013;6(1):92-101. February 2013.

43. DeFries R, Achard F, Brown S, Herold M, Murdiyarso D, Schlamandinger B, et al. Earth observation for estimating greenhouse gas emissions from deforestation in developing countries. Environ Sci Pol. 2007;10:385-94.

44. Saatchi S, Houghton RA, Dos Santos Alvala RC, Soares JV, Yu Y. Distribution of aboveground live biomass in the Amazon basin. Glob Chang Biol. 2007;13:813-37.

45. Karjalainen M, Kankare V, Vastaranta M, Holopainen M, Hyyppä J. Prediction of plot-level forest variables using TerraSAR-X stereo SAR data. Remote Sens Environ. 2012;117:338-47.

46. Asner GP, Mascaro J, Muller-Landau HC, Vieilledent G, Vaudry R, Rasamoelina M, et al. A universal airborne LiDAR approach for tropical forest carbon mapping. Oecologia. 2012;168:1147-60.

47. Asner GP, Mascaro J. Mapping tropical forest carbon: calibrating plot estimates to a simple LiDAR metric. Remote Sens Environ. 2014;140:614-24

48. d'Oliveiraa MVN, Reutebuch SE, McGaughey RJ, Andersen H-E. Estimating forest biomass and identifying low-intensity logging areas using airborne scanning lidar in Antimary State Forest, Acre State, Western Brazilian Amazon. Remote Sens Environ. 2012;124: 479-91.

49. Zolkos SG, Goetz SJ, Dubayah R. A meta-analysis of terrestrial aboveground biomass estimation using lidar remote sensing. Remote Sens Environ. 2013;128(2013):289-98.

50. Asner GP. Tropical forest carbon assessment: integrating satellite and airborne mapping approaches. Environ Res Let. 2009;4(n.3): 034009 . 
51. Wulder MA, White JC, Nelson RF, Naesset E, Ørka HO, Coops $\mathrm{NC}$, et al. Lidar sampling for large-area forest characterization: a review. Remote Sens Environ. 2012;121(2012):196-209.

52. Zeide B. Plot size optimization. For Sci. 1980;26:251-7.

53. Rahlf J, Breidenbach J, Solberg S, Naesset E, Astrup R. Comparison of four types of 3D data for timber volume estimation. Remote Sens Environ. 2014;155(2014):325-33.

54. Asner GP, Kellerc M, Pereira R, Zweeded JC. 2002. Remote sensing of selective logging in Amazonia Assessing limitations based on detailed field observations, Landsat ETM+, and textural analysis. Remote Sens Environ. 2002;80:483-96.

55. Asner GP, Knapp DE, Broadbent EN, Oliveira PJC, Keller M, Silva JN. Selective logging in the Brazilian Amazon. Science. 2005;310(5747):480-4.

56. De Sy V, Herold M, Achard F, Asner GP, Held A, Kellndorfer J, et al. Synergies of multiple remote sensing data sources for REDD+ monitoring. Curr Opin Environ Sustainab. 2012;4(6):696-706.

57. Achard F, Beuchle R, Mayaux P, Stibig H-J, Bodart C, Brink A. Determination of tropical deforestation rates and related carbon losses from 1990 to 2010. Glob Chang Biol. 2014;20(2014): 2540-54.

58. DeVries B, Verbesselt J, Kooistra L, Herold M. Robust monitoring of small-scale forest disturbances in a tropical montane forest using Landsat time series. Remote Sens Environ. 2015;161(May 2015): $107-21$.

59.• Tewkesbury AP, Comber AJ, Tate NJ, Lamb A, Fisher PF. A critical synthesis of remotely sensed optical image change detection techniques. Review article. Remote Sens Environ. 2015;160:1-14. Providing critical review to change detection.

$60 . \bullet$ Hansen M, Potapov P, Moore R. High-resolution global maps of 21st-century forest cover change. Science. 2013;134(2013):20114. High quality global example for Redd+type of applications.

61. Morales-Barquero L, Skutsch M, Jardel-Peláez EJ, Ghilardi A, Kleinn C, Healey JR. Operationalizing the definition of forest degradation for REDD+, with application to Mexico. Forests. 2014;5: 1653-81. doi:10.3390/f5071653.

62. Reiche J, Verbesselt J, Hoekman D, Herold M. Fusing Landsat and SAR time series to detect deforestation in the tropics. Remote Sens Environ. 2015;156:276-93. January 2015.

63. Tokola T, Hou Z. Alternative remote sensing materials and inventory strategies in tropical forest inventory. Case Lao PDR Ambiência. 2012;8(4):483-500.

64. McKeana J. Roering J (2004) Objective landslide detection and surface morphology mapping using high-resolution airborne laser altimetry. Geomorphology. 2003;1412:1-21.

65. Nelson RF, Hyde P, Johnson P, Emessiene B, Imhoff ML, Campbell $\mathrm{R}$, et al. Investigating RaDAR-LiDAR synergy in a North Carolina pine forest. Remote Sens Environ. 2007;110:98-108.

66. Olofsson P, Foody GM, Herold M, Stehman SV, Woodcock CE, Wulder MA. Good practices for estimating area and assessing accuracy of land change. Remote Sens Environ. 2014;148(2014):4257.

67. McRoberts RE. Satellite image maps: scientific inference of pretty pictures? Remote Sen Environ. 2011;115:715-24. 